\title{
Adolescents' Reproductive Health Status In Urban Slums In The Khon Kaen Municipality, Thailand
}

Soiy Anusornteerakul, Khon Kaen University, Thailand Pakvilai Srisaeng, Khon Kaen University, Thailand

Nilubon Rujiraprasert, Khon Kaen University, Thailand Somjit Maungpin, Khon Kaen University, Thailand

\begin{abstract}
This descriptive study aimed to explain the reproductive health of urban slum adolescents in the Khon Kaen Municipality area of Khon Kaen, Thailand. A self-reported questionnaire that took about 20 minutes to complete was used for data collection. Multi-stage simple random sampling was adopted in the selection of five target communities to recruit 277 male and female adolescents aged 10-19 years in accordance with the proportion of male and female adolescents in the area. Frequencies, percentages, standard deviations, and means were used for the data analysis.

The female and male participants had an average age of $14.62 \pm 2.66$ years and $14.58 \pm 2.84$ years, respectively. The average menarcheal age was $12.96 \pm 1.58$ years, while the age at which the first wet dream was experienced for boys was $14.12 \pm 1.44$ years. Most of the participants were in elementary school, while $5.7 \%$ of female and $2.4 \%$ of male adolescents did not attend school. Most girls and boys knew about contraceptive pills and condoms, but not other birth control methods. Contraceptive pills were used by $26.4 \%$ of female adolescents and condoms were used by $39.8 \%$ of male adolescents. However, it was reported that less than $10 \%$ of males and females regularly used condoms.

The lowest age of the $36.5 \%$ of girls who had had a sexual experience was 10 years, whereas the lowest age of the 40.7\% of sexually experienced boys was 11 years. The average age of the group of females who had started to have sexual intercourse was $14.81 \pm 1.71$ years, and the average age of the group of males who had had sexual intercourse was $15.23 \pm 1.32$ years. Most of the sample had had intercourse with their girlfriends or boyfriends. It was found that $1.3 \%$ of the girls and $1.8 \%$ of the boys were prostitutes and that $10.9 \%$ of the boys had visited brothels. Twenty-two percent of the girls admitted that they had masturbated, while $41.0 \%$ of the males did. About $17.8 \%$ of the female adolescents had been pregnant; $50 \%$ of those pregnancies ended in abortion and $50 \%$ of the females had been pregnant more than once.
\end{abstract}

Keywords: Adolescent; Reproductive Health; Urban Slum; Thailand

\section{INTRODUCTION}

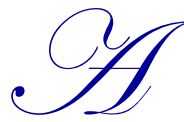

dolescents in the slum areas are considered minorities with low incomes. Most of these adolescents are from families with multiple problems and are likely to engage in risky sexual behavior, early sexual initiation, and unplanned pregnancy. A report from another country showed a high incidence of pregnancy among adolescents; four out of 10 became pregnant before 20 years of age and had been infected with Chlamydia and Gonorrhea (Lowdermilk, Perry \& Cashion, 2010). Around the world, 10-60\% of adolescents have unwanted pregnancies and as many as 4 million unsafe abortions are conducted yearly (WHO, 2010a). In Thailand, a survey was conducted on the sexual behaviors of 2,301 Grade 12 students from six schools who were randomly selected across economic statuses, religions, and housing types. It was found that $43 \%$ of girls aged under 18 years 
old had had sexual relationships, of which $20 \%$ were reported to have involved forced intercourse and $25 \%$ of them got pregnant (Vuttanont et al., 2006). Some of these adolescents chose to have abortions, a reproductive issue that could cause negative psychological and physiological impacts for both their families and public health services (WHO, 2010b).

Reproductive health is a factor indicating a population's quality of life. Slum adolescents are most vulnerable since they live in a community that lacks sufficient resources. Their families have to work hard but earn low incomes, have low levels of education, and have less access to health services. All of these factors make their lives quite different from others who live in better environments in terms of lifestyle, beliefs, health, and reproductive health. A study conducted on the reproductive health conditions of 525 male and female adolescents in the Klong Toey slum community in Bangkok, whose average age was $15 \pm 2.8$ years and more than $78 \%$ were still in school, showed that $13.0 \%$ of adolescents had had sexual experiences, with $4 \%$ of them reporting unwanted pregnancies. About $14 \%$ of these adolescents reported using medication to end their pregnancies. As expected, the results also showed that female adolescents had only a medium level of reproductive health knowledge and received little reproductive health information (Charanasri et al., 2004). This study showed that both male and female adolescents in slum communities are at risk of reproductive health problems. However, few studies have been conducted on their reproductive health even though these adolescents are in an underprivileged group in terms of both opportunities and quality of life. A research study on the sexual behaviors of teenagers in the social and cultural contexts revealed that different beliefs, values, and cultures lead to different behaviors among adolescents (Crombleohlm, 2009) and that different living conditions and environments can lead to different behaviors (Angstman et al., 2007; Niederhauser \& Markowitz, 2007). Socio-cultural factors are important in the planning of reproductive care and services for slum adolescents (Pillitteri, 2010). This study, therefore, aimed at investigating the reproductive health conditions of vulnerable male and female adolescents who live in slum areas in the Khon Kaen Municipality in Khon Kaen. The place was selected because of its location in the Northeast, which has a specific social and cultural identity (Maungpin et al., 2010). The results will provide valuable data for prevention planning with respect to the reproductive health problems of adolescents in slum areas in the Northeast.

\section{METHODOLOGY}

Descriptive research was employed in this study and the data were collected by means of a structured questionnaire completed in roughly 20 minutes by the participants themselves. The population under study included 10- to 19-year-old male and female adolescents living in 12 slum communities in the Khon Kaen Municipality in the Khon Kaen Province. A simple random method was carried out in a total of 88 slum communities (Department of Social Welfare, 2010) and the survey was conducted from January to April 2011 using the following steps:

1. The study sites were determined only within the crowded living areas around the Khon Kaen Municipality in Khon Kaen, which is the center of northeastern Thailand, where many slum communities are located. The sizes of these slums differ, but they are scattered around the area under the Khon Kaen Municipality.

2. There were 992 adolescents aged 10-19 years in 12 slum communities. The inclusion criterion was their living in the communities for a period of at least one year before the study was conducted. The sample size was determined by Cochran's (1977) sampling size formula for survey research. The proportion of the sampling group was decided at the population proportion $\mathrm{P}=0.05$, a reliability of $95 \%(\mathrm{Z})$, and acceptable discrepancies (d) of 5\%. The number derived for the sample size was 277.

3. Multi-stage simple random sampling without replacement was then performed; five of the 12 communities were sampled. There were 583 adolescents aged between 10-19 years, 248 male and 335 female. Next, simple random sampling was conducted according to the population proportion of the five communities, and 277 adolescents were recruited (Table 1). 
Table 1: The Proportion Of Participants Divided By Community And Sex

\begin{tabular}{|l|c|c|c|c|c|}
\hline \multirow{2}{*}{ Communities } & \multicolumn{2}{|c|}{ Males } & \multicolumn{2}{c|}{ Females } & \multirow{2}{*}{ Total Sample } \\
\cline { 2 - 6 } & Total & Sample & Total & Sample & 30 \\
\hline 1. Community 1 & 21 & 10 & 42 & 20 & 27 \\
\hline 2. Community 2 & 17 & 8 & 40 & 19 & 53 \\
\hline 3. Community 3 & 29 & 14 & 82 & 39 & 83 \\
\hline 4. Community 4 & 99 & 47 & 76 & 36 & 84 \\
\hline 5. Community 5 & 82 & 39 & 95 & 45 & $\mathbf{2 7 7}$ \\
\hline Total & $\mathbf{2 4 8}$ & $\mathbf{1 1 8}$ & $\mathbf{3 3 5}$ & $\mathbf{1 5 9}$ & \\
\hline
\end{tabular}

A structured questionnaire was used as the research tool. It consisted of general information of the sample groups and their reproductive health condition. The researchers tested the questionnaire beforehand to check the validity and the clarity of the language used. The test was conducted with 20 slum adolescents and yielded a Cronbach's alpha coefficient of 0.93 . Then the participants individually answered the questionnaire distributed at their homes after their parents were informed of the research objectives, methods of data collection, research benefits, protection of human rights, and confidentiality if they gave their oral consent to the survey. The researchers asked the parents to allow the participants to complete the questionnaire privately. The questionnaire was collected immediately upon completion. The analysis was performed using frequencies, percentages, standard deviations, and means.

\section{Ethics And Protection Of The Respondents' Rights}

Research related to sexuality and sexual behaviors is especially sensitive and may lead to negative feelings, especially among female adolescents. The researchers were aware that adolescents are timid and may not want to reveal some of their sexuality and sexual behaviors. Therefore, the respondents' rights were protected and the participants could have refused to answer any of the questions or withdraw from the study at any time if they wished to do so. Research ethics and morals were taken into account and the research itself was approved by the Human Research Ethics Committee of Khon Kaen University based on the referenced document HE 532092/ 2010 dated 26 May, 2010.

\section{RESULTS}

\section{Characteristics of the Participants}

The 277 participants included 118 males (42.6\%) and 159 females (57.4\%). Some of the participants did not answer some questions. The proportions of male and female adolescents in the age groups between 10 and 14 years old and 15 and 19 years old were more or less equal. The females' average age was $14.62 \pm 2.66$ years and the average age of first menstruation was $12.96 \pm 1.58$ years. The average age of the male adolescents was $14.58 \pm 2.84$ years and the average age of their first wet dream was $14.12 \pm 1.44$ years. These findings indicated that females entered adolescence about two years earlier than males.

The highest education of both males and females was the primary level, at $44.0 \%$ and $50.0 \%$, respectively. About $5.7 \%$ of females did not attend school, compared to $2.4 \%$ of males, indicating more illiteracy among female adolescents. More female adolescents attended vocational schools at the certificate and diploma levels than male adolescents. However, more males continued on to secondary and high schools than females.

\section{Birth Control}

About $63 \%$ of the female adolescents knew about contraceptive pills, whereas $70.3 \%$ of the male adolescents did not know about them. Only $4.4 \%$ to $39.0 \%$ of female adolescents, compared to only $0.8 \%$ to $18.6 \%$ of males, knew about emergency birth control pills, contraceptive injections, implant contraceptives, intra-uterine devices, misoprostol, the fertility awareness method, coitus interrupts, or vaginal douche. Both males (67.8\%) and females $(56.0 \%)$ knew about condoms. 
When asked about their experience using contraceptives, $26.4 \%$ of the females reported that they had used contraceptive pills, $8.1 \%$ had used contraceptive injections, and $23.9 \%$ had used condoms. Among the male adolescents, $39.8 \%$ had used condoms, but only $10 \%$ had used them regularly; $4.7 \%$ had used the fertility awareness method; and $11.2 \%$ had used the withdrawal method.

\section{Sexual Behaviors and Pregnancy}

Fifty percent of both male and female adolescents had a boyfriend or a girlfriend. About $11.3 \%$ of females reported that they had lived with their boyfriends without being marriage, 4.5 times higher than male adolescents of whom only $2.5 \%$ were living with their girlfriends. It should be noted that $3.1 \%$ of female adolescents marked their marital status as divorced.

Forty-one percent of male adolescents and $22.2 \%$ of females reported having masturbated. The age at the first sexual experience for female and male adolescents was 10 years and 11 years, respectively. Overall, the adolescents in the sample had a median age at first sexual intercourse of $14.81 \pm 1.71$ years, with females at $14.46 \pm$ 1.91 years and males at $15.23 \pm 1.32$ years. It can be seen that girls started to have sexual intercourse about one year earlier than boys. Twice as many females (15.8\%) started to have sexual relationships between 10 and 14 years of age. Among all the participants, $36.5 \%$ of females and $40.7 \%$ of males had sexual intercourse. All 58 female adolescents who had sexual relations $(100 \%)$ did it with their boyfriends, whereas $93.7 \%$ of the males had sex with their girlfriends. Among the 58 female adolescents who had sexual intercourse, 28 became pregnant (48.3\%). Among this number, 50\% reported having been pregnant more than once. Fourteen of the 28 girls who got pregnant resorted to abortion $(50 \%)$.

About $10.5 \%$ of females and $17.2 \%$ of males reported having more than one partner at the same time, with $6.9 \%$ of females and $8.5 \%$ of males stating that they had had more than three sexual partners. The highest number of sex partners for males and females in this sample was seven and six, respectively. In addition, $10.9 \%$ of the male adolescents reported having visited brothels. It was found that $1.3 \%$ of the female and $1.8 \%$ of the male adolescents were prostitutes.

\section{DISCUSSION AND CONCLUSION}

\section{Characteristics of the Participants}

Twice as many female adolescents as males in slums did not go to school. Gender inequality (WHO, 2008) may also contribute to different levels of access to education between male and female adolescents, as girls are often pulled out of school and boys left in school when family income dictates that all the children cannot be educated. Moreover, the fact that girls get married early makes it difficult for them to continue their education, as it is more complicated for females to remain in school or continue their education than males once they get married. Once out of school, these adolescents are likely to engage in early sexual relations and are at increased risk for unplanned pregnancy or other risky sexual behaviors (Lowdermilk, Perry, \& Cashion, 2010).

For male adolescents, marriage had little effect on their education. They could continue their study more easily than the girls (Sultana, 2005). A study in Pakistan in 2005 similarly showed that males had a greater chance than females to continue their education. They also found that the age of marriage was correlated to education. Loweducated or illiterate women get married at the age of 20 years or less (Sultana, 2005). The fact that more girls went to vocational schools at both the certificate and diploma levels than boys and that more boys continued in general education shows that the girls had less opportunity to continue on to the high school level. Girls are more likely to be trained in vocations, which take less time to learn and pay less.

\section{Birth Control}

The female adolescents knew about many types of contraceptives, including pills, injections, Norplant, and intra-uterine devices, as these are the contraceptive types used by females, while most males knew only about condoms. The results indicated that adolescents learned about contraceptives through direct experience. These 
findings correlated to a study conducted by Touko et al. (2004) on 1,520 male and female teenagers aged 10 to 19 years who lived in urban and rural areas of Cameroon. It was found that female adolescents were better informed regarding different types of family planning methods than males and those males (82\%) knew about condoms more than females at a significant level. The fertility awareness method and coitus interrupts were found to be practiced by many adolescents. Our findings indicated a very low level of regular birth control and condom use because most adolescents were in primary school, where contraceptives were not taught, and they came from low-income families with low levels of education. Therefore, their level of access to health information and contraception services was low. Consequently, the perception of pregnancy risk and information related to pregnancy prevention was low (Charanasri et al., 2004).

\section{Sexual Behaviors and Pregnancy}

More female than male adolescents had steady partners, so they married earlier. Similarly, a study in Pakistan showed that females got married two to three years earlier than males (Sultana, 2005). In our study, most of the adolescents in the sample groups were students; therefore, most of them were single. The proportion of females living with their partners before marriage was higher than that of males. Divorce/separation rates, which may occur even when the women were still pregnant, was higher in females than those of males (3.1\%). When this happens, it is typical in Thai society that a mother is the primary caretaker for the babies. The young mothers and her partners were forced to leave their children with their parents while they were out working to make a living, having very low incomes in most cases. Raising a child is difficult when both parents are working, especially for adolescent parents, which can lead to divorce and other serious problems, including domestic violence and child abuse.

As high as $36.5 \%$ of female adolescents and $40.7 \%$ of male adolescents were sexually active. The study showed that the respondents of both sexes had sexual relations at a very young age or at school age. This is because most slum adolescents leave school at an early age and live in a socio-cultural context where early marriage is common. Social context and beliefs dictate people's roles and behaviors (Whitley \& Kirmayer, 2008). The average age of female adolescents at first intercourse was 14.5 years, while that of male adolescents was 15.2 years, which is lower than the average age at first intercourse of Thai teenagers (16.5 years), in general, and lower than those of teenagers in other countries, including Canada and the U.S. (15 years), England (15.3 years), Germany (15.6 years), France (15.8 years), and Taiwan (17 years) (London International Group PLC, 2009).

The highest number of sexual partners among the adolescents was six for the females and seven for the males. This shows that having more than one sexual partner is common among Thai adolescents, yielding the highest infidelity rate in the world (London International Group PLC, 2009). This study found that nearly all male and female adolescents had had sex with their boyfriends or girlfriends. Female adolescents were found to have had sex with boyfriends or friends more frequently than male adolescents, partly because males bought commercial sex or were commercial sex workers themselves.

Both males and females showed a rather high level of masturbation, higher than that of urban slum adolescents in India, where only $3.18 \%$ of males and $2.7 \%$ of females were found to have masturbated (Watsa, 2003). Our findings indicated that $48.3 \%$ of the girls who had had sexual relations became pregnant, accounting for $17.6 \%$ of all 159 female participants - higher than the $10 \%$ criterion stated by the World Health Organization (WHO, 2010a). This result showed that the adolescents lacked awareness and probably knowledge and understanding of sex education and the prevention of pregnancy.

\section{SUGGESTIONS}

The prevention of unwanted pregnancies among adolescents should involve multi-sectoral collaboration involving public health officers, teachers, families, communities, and friends, as the problem of pregnancy and risky sexual behavior cannot be solved with one strategic approach (Anusornteerakul et al., 2008; Greenberg et al., 2003). Suggestions for prevention of pregnancy include:

1. This study indicated that a high proportion of adolescents became pregnant during their school years, so there is a need to consider when and how sex education should be integrated into the academic curriculum 
so that adolescents will be equipped with pregnancy prevention knowledge before they turn 10-11 years old, which is the youngest age at first intercourse for male and female adolescents. Sexuality education should emphasize not only abstinence, but also contraceptives, to prevent pregnancy and sexually transmitted infections.

2. Vulnerable adolescents in slums have less chance of continuing their education; therefore, approaches to solve pregnancy and STIs should also focus on adolescents who are not enrolled in school. Parent-based approaches should be encouraged as an effective strategy to solve the problem of unwanted pregnancy and unsafe abortion, as studies have shown that strong relationships between children and parents directly deter the first sexual experiences among children (Sieving, McNeely, \& Blum, 2000) and that parents should be good role models in terms of sex. This can be achieved by informing parents of sex education, birth control, adolescent development, adolescent context, and effective communication skills which, in turn, will enable the parents to show their adolescent children love and support. Parents should be prepared to provide correct information with supportive attitudes. They should be sensitive to adolescents' problems. This will also enhance family relationships and reduce conflicts between adolescents and their parents.

3. Society should enable communities to see the importance of education because, if adolescents remain in school, their chances of having sex and getting married will decrease while their access to reproductive health information and services will increase. However, most adolescents in slums are from families with low incomes. Therefore, the local and national governments should improve their access to higher education through offering scholarships. Families should be made aware of the importance of their children's education. Special scholarships, with the condition that the adolescents will return to work in their communities after graduation, may be one of the strategies employed to continue to improve the lives of adolescents and the community environment. Additionally, a youth-friendly reproductive health service unit should be set up in communities so that the adolescents can conveniently and easily access information and services when they feel they need them.

\section{AUTHOR INFORMATION}

Soiy Anusornteerakul is Associate Professor in nursing and corresponding author, currently working at WHO Collaboration Center for Research and Training on Gender and Women's health, Faculty of Nursing, Khon Kaen University, Thailand. She finished her doctoral degree in Development Sciences from Faculty of Humanities and Social Sciences, Khon Kaen University, Thailand. Most of her research focuses on female and adolescent reproductive health. E-mail: soianu@kku.ac.th (Corresponding author)

Pakvilai Srisaeng is Assistant Professor in nursing, currently working at WHO Collaboration Center for Research and Training on Gender and Women's Health, Faculty of Nursing, Khon Kaen University, Thailand. She finished her doctoral degree in Nursing from Case Western Reserve University, Cleveland, Ohio, USA. Most of her research focuses on female reproductive health and depression. E-mail: spakvi@kku.ac.th

Nilubon Rujiraprasert is Assistant Professor in nursing, currently working at WHO Collaboration Center for Research and Training on Gender and Women's Health, Faculty of Nursing, Khon Kaen University, Thailand. She finished her doctoral degree in Nursing from Chiangmai University, Thailand. Most of her research focuses on adolescent reproductive health. E-mail: nilruj@kku.ac.th

Somjit Maungpin is lecturer in nursing, currently working at WHO Collaboration Center for Research and Training on Gender and Women's Health, Faculty of Nursing, Khon Kaen University, Thailand. She finished her doctoral degree in Nursing from Chiangmai University, Thailand. Most of her research focuses on adolescent pregnancy. E-mail: sompha@kku.ac.th

\section{REFERENCES}

1. Angstman, S., et al. (2007). Tobacco and other substance use among Alaska native youth in Western Alaska. American Journal of Health Behavior, 31(3) 249-260. 
2. Anusornteerakul, S., Khamnarong, K., Khamanarong, S., \& Thinkhamrop, J. (2008). The influence factors that affect Thailand's management of youth reproductive health service. Journal of Diversity Management, 3(4) 27-32.

3. Department of Social Welfare. (2010). Reporting data of Khon Kaen community in 2009. Khon Kaen: The Council of Khon Kaen Municipal Affairs Office.

4. Charanasri, C., Padumanonda, S., Boonmongkon, P., Imamee, N., \& Pandii. W. (2004). Reproductive health status and reproductive health care behavior of female adolescents in over populated communities, Bangkok Metropolis. Journal of Health Science, 13(6) 958-968.

5. Crombleholme, W.R. (2009). Obstetrics. In S. J. Mcphee \& M. A. Papadakis. (Eds). Current medical diagnosis \& treatment. Columbus, IH: McGraw-Hill.

6. Cochran, W.G. (1977). Sampling techniques. $3^{\text {rd }}$ ed. Canada: John Wiley \& Sons.

7. Greenberg, M.T., Weissberg, R.P., O’Brien, M.T., Zines, J.E., Fredericks, L., Resnik, H., \& Elias, M.J. (2003). Enhancing school based prevention and youth development through coordinated social, emotional and academic learning. American Psychologist, 58(6-7) 466-474.

8. London International Group PLC. (2009). Durex global sex survey 1999--the perspective of teenagers 2009. Retrieved from http:// www.samunpai.com/sex/show.php. Available at September 18, 2009.

9. Lowdermilk, D.L., Perry, S.E., \& Cashion, K. (2010). Maternity Nursing. $8^{\text {th }}$ ed. Canada: Mosby.

10. Muangpin, S., et al. (2010). Northeastern Thai adolescents' perceptions of being unmarried and pregnant. Pacific Rim Int J Nurs Res, 14(2) 149-161.

11. Niederhauser, V.P., \& Markowitz, M. (2007). Barriers to immunizations: multiethnic parents of under and unimmunized children speak. Journal of the American Academy of Nurse Practitioner, 19(1), 15-23.

12. Pillitteri, A. (2010). Maternal \& child health nursing: care of the childbearing \& childrearing family. $6^{\text {th }}$ ed. China: Lippincott William \& wilkins.

13. Prohmmo, A. (2007). Adolescent health and development situation in Thailand. Naknon Prathom: Institute for Population and Social Research, Mahidol University.

14. Sieving, R., McNeely, C.G., \& Blum, R.W. (2000). Maternal expectation, mother-child connectedness, and adolescent sexual debut. Archives-Based Healthcare, 7(1) 14-17.

15. Sultana, M. (2005). Adolescents and youth in Pakistan: culture of silence. UK: Department for International Development.

16. Touko, A., Kemegne, J., Kiawi, E., \& Lamien, B. (2004). Gender differentials in adolescents' reproductive health behavior. Int Conf AIDs, 15(Jul) 11-16.

17. Vuttanont, U., Greenhagh, T., Griffin, M., \& Boynton, P. (2006). Sweet boys and sweet girls, education needs in Thai teenagers: a mixed-method study. Lancet, 368, 2068-80.

18. Watsa, M.C. (2003). Adolescent sexuality - an unexplored territory. Thai J Health Res, 17(2) 86-97.

19. Whitley, R., Kirmayer, L.J. (2008). Perceived stigmatization of young mothers: an exploratory study of psychological and social experience. Social Science and Medicine, 66(2) 337-348.

20. World Health Organization. (2010a). Pregnancy adolescent: delivering on global promise of hope. Retrieved from http://whglibdoe.who.int/publications.cited 20 march 2010.

21. World Health Organization. (2010b). Complications for abortion: technical and managerial guidelines for prevention and treatment. Retrieved from http://whglibdoe.who.int/publications.cited 28 march 2010.

22. World Health Organization. (2008). Gender and health reference manual. Nepal Government: Ministry of Health and Population and Tribhuvan University. 
NOTES 